\title{
Non contiguous-finished genome sequence and description of Peptoniphilus grossensis sp. nov.
}

\author{
Ajay Kumar Mishra, Perrine Hugon, Catherine Robert, Didier Raoult and Pierre-Edouard \\ Fournier \\ Unité de Recherche sur les Maladies Infectieuses et Tropicales Emergentes, UMR CNRS \\ 7278
}

*Corresponding author: Pierre-Edouard Fournier (pierre-edouard.fournier@univmed.fr)

Keywords: Peptoniphilus grossensis, genome

Peptoniphilus grossensis strain ph $5^{\top} \mathrm{sp}$. nov., is the type strain of Peptoniphilus grossensis sp. nov., a new species within the Peptoniphilus genus. This strain, whose genome is described here, was isolated from the fecal flora of a 26-year-old woman suffering from morbid obesity. $P$. grossensis strain ph5 is a Gram-positive obligate anaerobic coccus. Here we describe the features of this organism, together with the complete genome sequence and annotation. The $2,101,866$-bp long genome (1 chromosome but no plasmid) exhibits a G+C content of 33.9\% and contains 2,041 protein-coding and 29 RNA genes, including 3 rRNA genes.

\section{Introduction}

Peptoniphilus grossensis strain ph5 $5^{\mathrm{T}}$ (= CSUR P184 = DSM 25475), is the type strain of Peptoniphilus grossensis sp. nov. This bacterium is a Grampositive, spore-forming, indole positive, anaerobic coccoid bacterium that was isolated from the stool of a 26-year-old woman suffering from morbid obesity.

Since 1995 and the sequencing of the first bacterial genome, that of Haemophilus influenzae, more than 3,000 bacterial genomes have been sequenced [1]. This was permitted by technical improvements as well as increased interest in having access to the complete genetic information encoded by bacteria. At the same time, biological tools for defining new bacterial species have not evolved, and DNA-DNA hybridization is still considered the gold standard [2] despite its drawbacks and the taxonomic revolution that has resulted from the comparison of $16 \mathrm{~S}$ rDNA sequences [3]. In this manuscript, we propose and describe a new Peptoniphilus species using genomic and phenotypic information [4] to.

Gram-positive anaerobic cocci (GPAC) are part of the commensal flora of humans and animals, and are also commonly associated with a variety of human infections [5,6]. Extensive taxonomic changes have occurred in this group of bacteria, especially in clinically-important genera such as Finegoldia, Micromonas, and Peptostreptococcus [7]. The genus Peptostreptococcus was divided into three genera: Peptoniphilus (Ezaki et al., 2001),
Anaerococcus (Ezaki et al., 2001) and Gallicola (Ezaki et al., 2001). The genus Peptoniphilus includes the following butyrate-producing, nonsaccharolytic species that use peptone and amino acids as major energy sources: $P$. asaccharolyticus, P. gorbachii, P. harei, P. indolicus, P. ivorii, $P$. lacrimalis [7], P. olsenii [8] and P. methioninivorax [9].

Members of the genus Peptoniphilus have mostly been isolated from various human clinical specimens such as vaginal discharges, ovarian, peritoneal, sacral and lacrymal gland abscesses [7]. In addition, $P$. indolicus causes summer mastitis in cattle [7].

Here we present a summary classification and a set of features for P. grossensis sp. nov. strain ph5 ${ }^{\mathrm{T}}$ (= CSUR P184 = DSM 25475), together with the description of the complete genomic sequencing and annotation. These characteristics support the circumscription of the species $P$. grossensis.

\section{Classification and features}

A stool sample was collected from a 26-year-old woman living in Marseille, France, who suffered from morbid obesity: BMI=48.2 $(118.8 \mathrm{~kg}, 1.57$ meter). At the time of stool sample collection, she was not a drug-user and was not on a diet. The patient gave an informed and signed consent, and the agreement of local ethics committee of the IFR48 (Marseille, France) were obtained under agreement 11-017. The fecal specimen was pre- 
served at $-80^{\circ} \mathrm{C}$ after collection. Strain $\mathrm{PH}^{\mathrm{T}}$ (Table 1) was isolated in 2011 by anaerobic cultivation on 5\% sheep blood-enriched Columbia agar (BioMerieux, Marcy l'Etoile, France) after 26 days of preincubation of the stool sample in rumen and ship blood bottle culture. This strain exhibited a 96.7\% nucleotide sequence similarity with $P$. harei and occupied an intermediate phylogenetic posi- tion between P. gorbachii and P. olsenii (Figure 1). Although sequence similarity of the $16 \mathrm{~S}$ operon is not uniform across taxa, this value was lower than the $98.7 \%$ 16S rRNA gene sequence threshold recommended by Stackebrandt and Ebers to delineate a new species without carrying out DNADNA hybridization [3].

Table 1. Classification and general features of Peptoniphilus grossensis strain ph5 ${ }^{\top}$ according to the MIGS recommendations [10]

\begin{tabular}{|c|c|c|c|}
\hline MIGS ID & Property & Term & Evidence code $^{\mathrm{a}}$ \\
\hline & Current classification & Domain Bacteria & TAS [11] \\
\hline & & Phylum Firmicutes & TAS [12-14] \\
\hline & & Class Clostridia & TAS $[15,16]$ \\
\hline & & Order Clostridiales & TAS $[17,18]$ \\
\hline & & Family Clostridiales family XI Incertae sedis & NAS \\
\hline & & Genus Peptoniphilus & TAS [7] \\
\hline & & Species Peptoniphilus grossensis & IDA \\
\hline & & Type strain: ph5 & IDA \\
\hline & Gram stain & Positive & IDA \\
\hline & Cell shape & Coccoid & IDA \\
\hline & Motility & Nonmotile & IDA \\
\hline & Sporulation & Sporulating & IDA \\
\hline & Temperature range & Mesophile & IDA \\
\hline & Optimum temperature & $37^{\circ} \mathrm{C}$ & IDA \\
\hline MIGS-6.3 & Salinity & growth in $\mathrm{BHI}$ medium $+5 \% \mathrm{NaCl}$ & IDA \\
\hline \multirow[t]{3}{*}{ MIGS-22 } & Oxygen requirement & Anaerobic & IDA \\
\hline & Carbon source & Unknown & \\
\hline & Energy source & Peptones & NAS \\
\hline MIGS-6 & Habitat & human gut & IDA \\
\hline MIGS-15 & Biotic relationship & free living & IDA \\
\hline \multirow[t]{3}{*}{ MIGS-14 } & Pathogenicity & Unknown & \\
\hline & Biosafety level & 2 & \\
\hline & Isolation & human feces & \\
\hline MIGS-4 & Geographic location & France & IDA \\
\hline MIGS-5 & Sample collection time & January 2011 & IDA \\
\hline MIGS-4.1 & Latitude & 43.296482 & IDA \\
\hline MIGS-4.2 & Longitude & 5.36978 & IDA \\
\hline MIGS-4.3 & Depth & Surface & IDA \\
\hline MIGS-4.4 & Altitude & $0 \mathrm{~m}$ above sea level & IDA \\
\hline
\end{tabular}

aEvidence codes - IDA: Inferred from Direct Assay; TAS: Traceable Author Statement (i.e., a direct report exists in the literature); NAS: Non-traceable Author Statement (i.e., not directly observed for the living, isolated sample, but based on a generally accepted property for the species, or anecdotal evidence). These evidence codes are from the Gene Ontology project [19]. If the evidence is IDA, then the property was directly observed for a live isolate by one of the authors or an expert mentioned in the acknowledgements. 


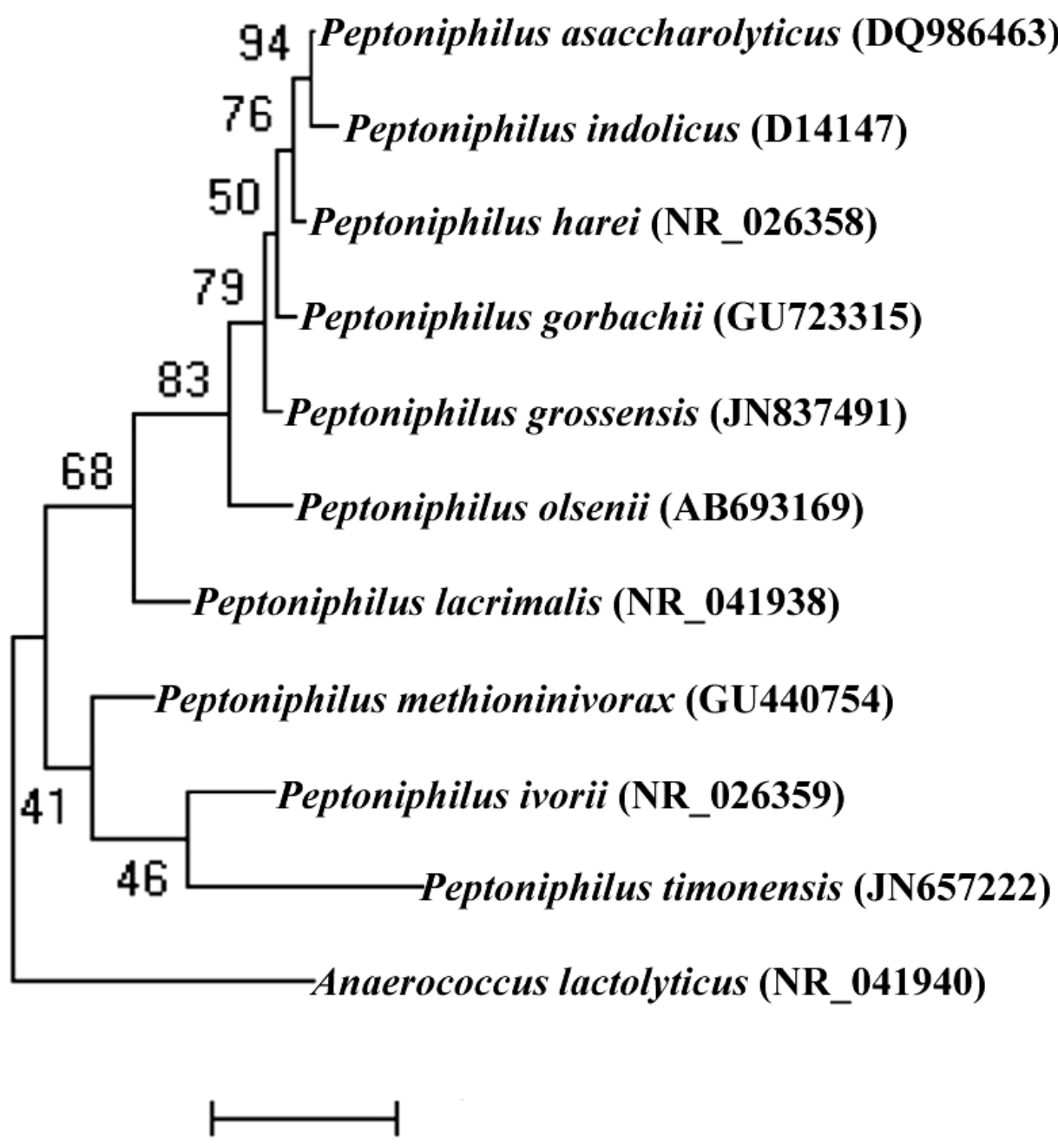

Figure 1. Phylogenetic tree highlighting the position of Peptoniphilus grossensis strain $p h 5^{\top}$ relative to other type strains within the Peptoniphilus genus. GenBank accession numbers are indicated in parentheses. Sequences were aligned using CLUSTALW, and phylogenetic inferences obtained using the maximumlikelihood method within MEGA program. Numbers at the nodes are percentages of bootstrap values obtained by repeating the analysis 500 times to generate a majority consensus tree. Anaerococcus lactolyticus was used as an outgroup. The scale bar represents a $2 \%$ nucleotide sequence divergence.

Different growth temperatures $\left(25,30,37,45^{\circ} \mathrm{C}\right)$ were tested; no growth occurred at $25^{\circ} \mathrm{C}, 30^{\circ} \mathrm{C}$ or $45^{\circ} \mathrm{C}$. Growth only occurred at $37^{\circ} \mathrm{C}$. Colonies were $2 \mathrm{~mm}$ in diameter on blood-enriched Columbia agar and Brain Heart Infusion (BHI) agar. Growth of the strain was tested under anaerobic and microaerophilic conditions using GENbag anaer and GENbag microaer systems, respectively (BioMérieux), and in the presence of air, with or without $5 \% \mathrm{CO}_{2}$. Growth was achieved only anaerobically. Gram staining showed Gram-positive cocci able to form spores (Figure 2). The motility test was negative. Cells grown on agar had a mean diameter of $1.2 \mu \mathrm{m}$ by electron microscopy and were mostly grouped in pairs, short chains or small clumps (Figure 3).

Strain ph5 exhibited neither catalase nor oxidase activities but indole production was observed. Using an API Rapid ID 32A strip (BioMerieux), a positive reaction was observed for Mannose fermentation, arginine arylamidase, tyrosine arylamidase, histidine arylamidase and leucine arylamidase. Strain ph5 was susceptible to penicillin G, amoxicillin, ceftriaxon, cefalexin, imipenem fosfomycin, erythromycin, doxycyclin, rifampin, vancomycin and metronidazole, but resistant to ciprofloxacin and cotrimoxazole. 


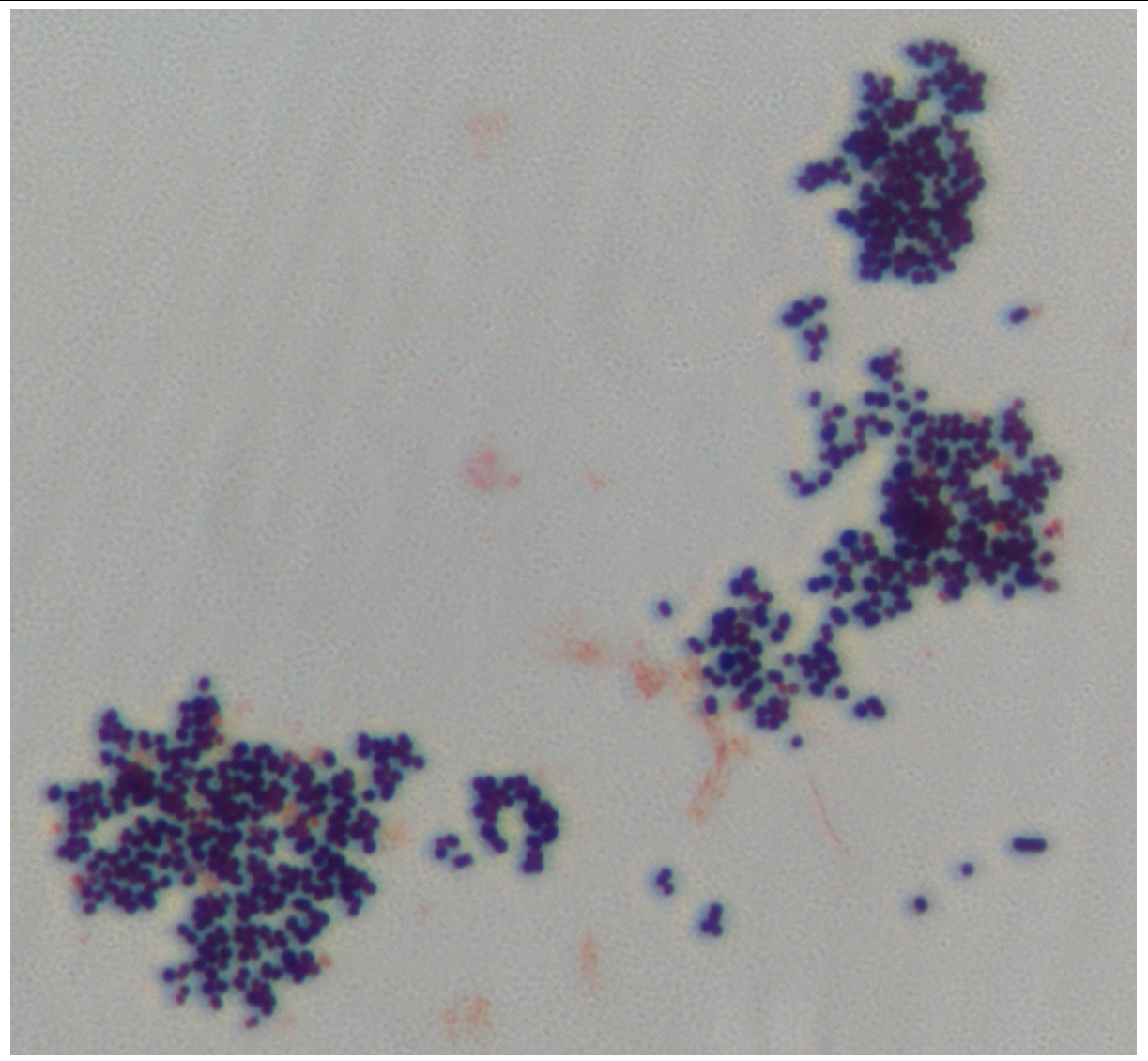

Figure 2. Gram staining of $P$. grossensis strain $p h 5^{\top}$

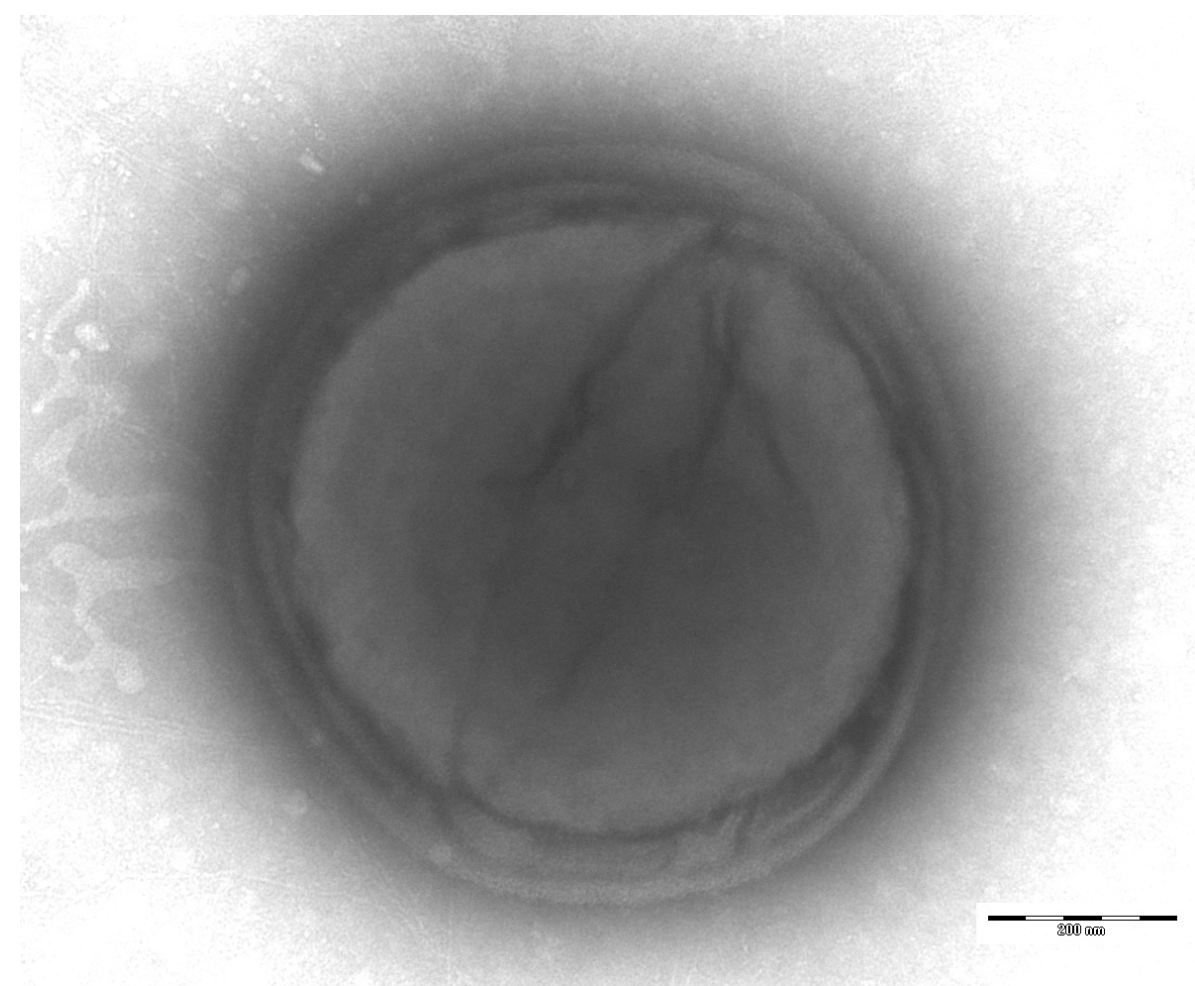

Figure 3. Transmission electron microscopy of $P$. grossensis strain ph $5^{\top}$, using a Morgani 268D (Philips) at an operating voltage of $60 \mathrm{kV}$. The scale bar represents $900 \mathrm{~nm}$. 
Matrix-assisted laser-desorption/ionization time-of-flight (MALDI-TOF) MS protein analysis was carried out as previously described [20]. Briefly, a pipette tip was used to pick one isolated bacterial colony from a culture agar plate and spread it as a thin film on a MTP 384 MALDI-TOF target plate (Bruker Daltonics, Germany). Twelve distinct deposits were done for strain ph5 from twelve isolated colonies. Each smear was overlaid with $2 \mu \mathrm{L}$ of matrix solution (saturated solution of alpha-cyano-4-hydroxycinnamic acid) in $50 \%$ acetonitrile, $2.5 \%$ tri-fluoracetic acid, and allowed to dry for five minutes. Measurements were performed with a Microflex spectrometer (Bruker). Spectra were recorded in the positive linear mode for the mass range of 2,000 to 20,000 Da (parameter settings: ion source 1 (ISI), $20 \mathrm{kV}$; IS2, $18.5 \mathrm{kV}$; lens, $7 \mathrm{kV}$ ). A spectrum was obtained after 675 shots at a variable laser power. The time of acquisition was between 30 seconds and 1 minute per spot. The twelve ph5 spectra were imported into the MALDI Bio Typer software (version 2.0, Bruker) and analyzed by standard pattern matching (with default parameter settings) against the main spectra of 3,769 bacteria, including spectra from 8 validated Peptoniphilus species used as reference data, in the Bio Typer database (updated March 15th, 2012). The method of identification includes the $\mathrm{m} / \mathrm{z}$ from 3,000 to $15,000 \mathrm{Da}$. For every spec- trum, 100 peaks at most were taken into account and compared with the spectra in database. A score enabled the presumptive identification and discrimination of the tested species from those in a database: a score $\geq 2$ with a validated species enabled the identification at the species level; a score $\geq 1.7$ but $<2$ enabled the identification at the genus level; and a score $<1.7$ did not enable any identification. For strain ph5, the obtained score was 1.3 , thus suggesting that our isolate was not a member of a known species. We incremented our database with the spectrum from strain ph5 (Figure 4).

\section{Genome sequencing information} Genome project history

The organism was selected for sequencing on the basis of its phylogenetic position and 16S rRNA similarity to other members of the genus Peptoniphilus. To date, the genomes from only three validated Peptoniphilus species have been sequenced. This was the first genome of Peptoniphilus grossensis sp. nov. A summary of the project information is shown in Table 2. The Genbank accession number is CAGX00000000 and consists of 77 contigs. Table 2 shows the project information and its association with MIGS version 2.0 compliance.

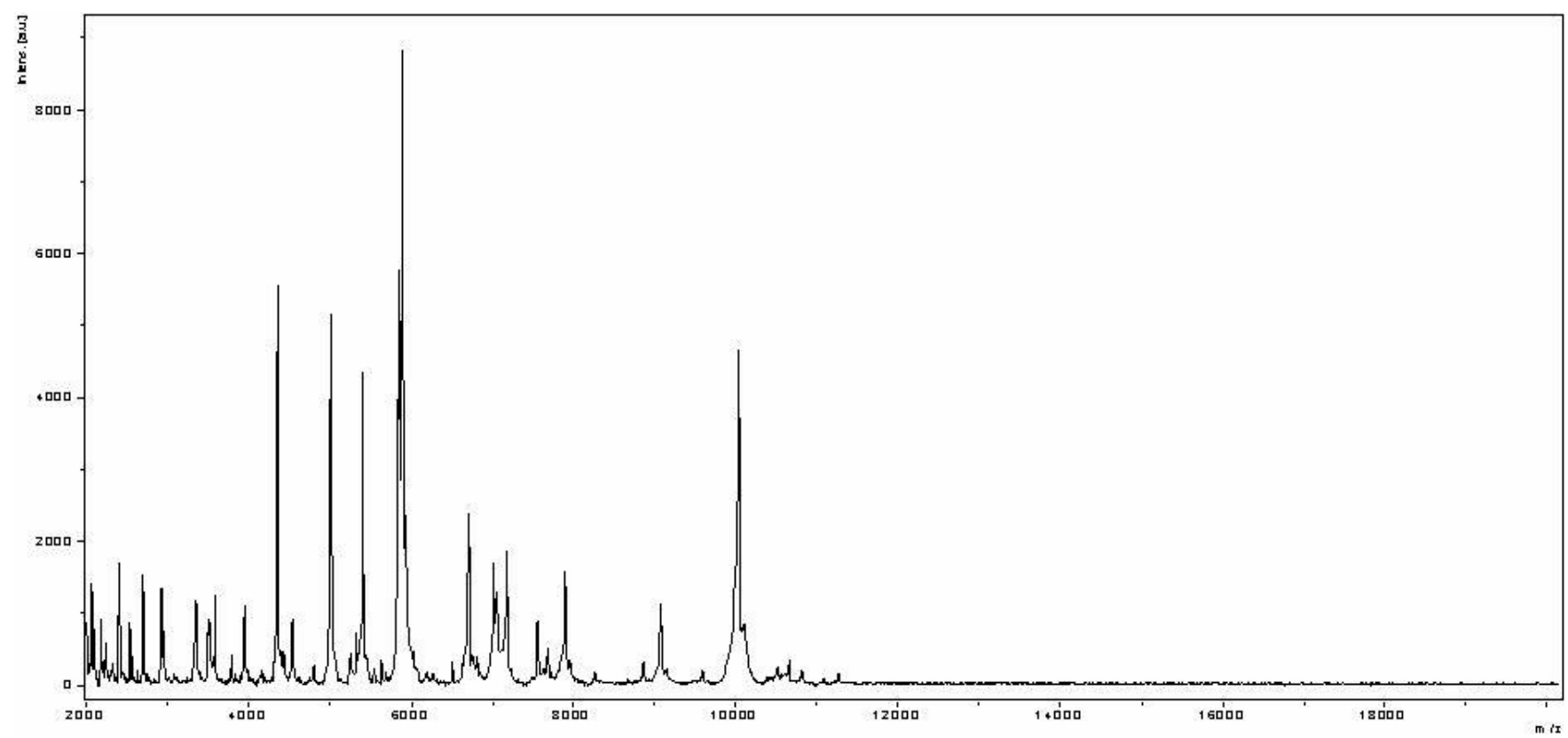

Figure 4. Reference mass spectrum from $P$. grossensis strain $p h 5^{\top}$. Spectra from 12 individual colonies were compared and a reference spectrum was generated. 
Table 2. Project information

\begin{tabular}{lll}
\hline MIGS ID & Property & Term \\
\hline MIGS-31 & Finishing quality & High-quality draft \\
MIGS-28 & Libraries used & One 454 paired end 3-kb library \\
MIGS-29 & Sequencing platforms & 454 GS FLX Titanium \\
MIGS-31.2 & Sequencing & 26.78× \\
MIGS-30 & Assemblers & Newbler version 2.5.3 \\
MIGS-32 & Gene calling method & Prodigal \\
& INSDC ID & PRJEB48 \\
& Genbank ID & CAGX00000000 \\
& Genbank Date of Re- & May 30, 2012 \\
& lease & Gi13722 \\
& Gold ID & Study of the human gut microbiome \\
\hline
\end{tabular}

\section{Growth conditions and DNA isolation}

P. grossensis strain ph5 ${ }^{\mathrm{T}}(=$ CSUR P184 $=\mathrm{DSM}$ 25475), was grown on blood agar medium at $37^{\circ} \mathrm{C}$. Six petri dishes were spread and resuspended in $6 \times 100 \mu$ l of G2 buffer (EZ1 DNA Tissue kit, Qiagen). A first mechanical lysis was performed by glass powder on the Fastprep-24 device (Sample Preparation system) from MP Biomedicals, USA) using 40 seconds cycles. DNA was then treated with $2.5 \mu \mathrm{g} / \mu \mathrm{L}$ lysozyme ( 30 minutes at $37^{\circ} \mathrm{C}$ ) and extracted through the BioRobot EZ 1 Advanced XL (Qiagen). The DNA was then concentrated and purified on a Qiamp kit (Qiagen). The yield and the concentration was measured by the Quant-it Picogreen kit (Invitrogen) on a Genios_Tecan fluorometer at $62 \mathrm{ng} / \mu \mathrm{l}$.

\section{Genome sequencing and assembly}

DNA $(5 \mu \mathrm{g})$ was mechanically fragmented on the Hydroshear device (Digilab, Holliston, MA, USA) with an enrichment size of 3-4kb. The DNA fragmentation was visualized using an Agilent 2100 BioAnalyzer on a DNA labchip 7500 to yield an optimal size of $3.16 \mathrm{~kb}$. The library was constructed according to the 454_Titanium paired-end protocol and manufacturer. Circularization and nebulization were performed and generated a pattern with an optimum at $628 \mathrm{bp}$. After PCR amplification through 15 cycles followed by double size selection, the single stranded paired end library was then quantified on the Quant-it Ribogreen kit (Invitrogen) on the Genios_Tecan fluorometer at $34 \mathrm{pg} / \mu \mathrm{L}$. The library concentration equivalence was calculated as $9.93 \mathrm{E}+08$ molecules $/ \mu \mathrm{L}$. The library was held at $-20^{\circ} \mathrm{C}$ until use.

The shotgun library was clonally amplified with 0.5 and $1 \mathrm{cpb}$ in 2 emPCR reactions per condition with the GS Titanium SV emPCR Kit (Lib-L) v2. The yields of the emPCR at $0.5 \mathrm{cpb}$ and $1 \mathrm{cpb}$ were of $9.63 \%$ and $22.35 \%$, respectively. A total of 790,000 beads for a $1 / 4$ region and 790,000 beads for a $1 / 8$ region were loaded on the GS Titanium PicoTiterPlates (PTP Kit $70 \times 75$ ) and sequenced with the GS Titanium Sequencing Kit XLR70.

The runs were performed overnight and then analyzed on the cluster through the gsRunBrowser and gsAssembler_Roche. The global 176,029 passed filter sequences generated $56.24 \mathrm{Mb}$ with a length average of $319 \mathrm{bp}$. These sequences were assembled using the Newbler software from Roche with $90 \%$ identity and $40 \mathrm{bp}$ as overlap. Seventy-seven large contigs ( $>1500 \mathrm{bp}$ ) were obtained, for a genome size of $2.1 \mathrm{Mb}$ which corresponds to a coverage of $26.78 \times$ genome equivalent.

\section{Genome annotation}

Open Reading Frames (ORFs) were predicted using Prodigal [21] with default parameters but the predicted ORFs were excluded if they were spanning a sequencing gap region. The predicted bacterial protein sequences were searched against the GenBank database [22] and the Clusters of Orthologous Groups (COG) databases using BLASTP. The tRNAScanSE tool [23] was used to find tRNA genes, whereas ribosomal RNAs were found by using RNAmmer [24] and BLASTn against the GenBank database. Lipoprotein signal peptides and numbers of transmembrane helices were predicted using SignalP [25] and TMHMM [26] respectively. ORFans were identified if their BLASTP $E$-value was lower than $1 \mathrm{e}-3$ for alignment length greater than 80 amino acids. If alignment lengths were smaller than 80 amino acids, we used an $E$-value of 1e-05. Such parameter thresholds have already been used in previous works to define ORFans. To 
estimate the mean level of nucleotide sequence similarity at the genome level between Peptoniphilus species, we compared the ORFs only using BLASTN and the following parameters: a query coverage of $\geq 70 \%$ and a minimum nucleotide length of $100 \mathrm{bp}$. Artemis [27] was used for data management and DNA Plotter [28] was used for visualization of genomic features. Mauve alignment tool was used for multiple genomic sequence alignment and visualization [29].

\section{Genome properties}

The genome of $P$. grossensis sp. nov. strain ph5 $5^{\mathrm{T}}$ is $2,101,866$ bp long ( 1 chromosome, but no plasmid) with a $33.9 \% \mathrm{G}+\mathrm{C}$ content of (Figure 5 and Table $3)$. Of the 2,070 predicted genes, 2,041 were protein-coding genes, and 29 were RNAs. Three rRNA genes (one 16S rRNA, one 23S rRNA and one 5S rRNA) and 26 predicted tRNA genes were identified in the genome. A total of 1,439 genes (69.52\%) were assigned a putative function. One hundred and fifty-five genes were identified as ORFans (7.6\%). The remaining genes were annotated as hypothetical proteins. The properties and statistics of the genome are summarized in Table 3. The distribution of genes into COGs functional categories is presented in Table 4.

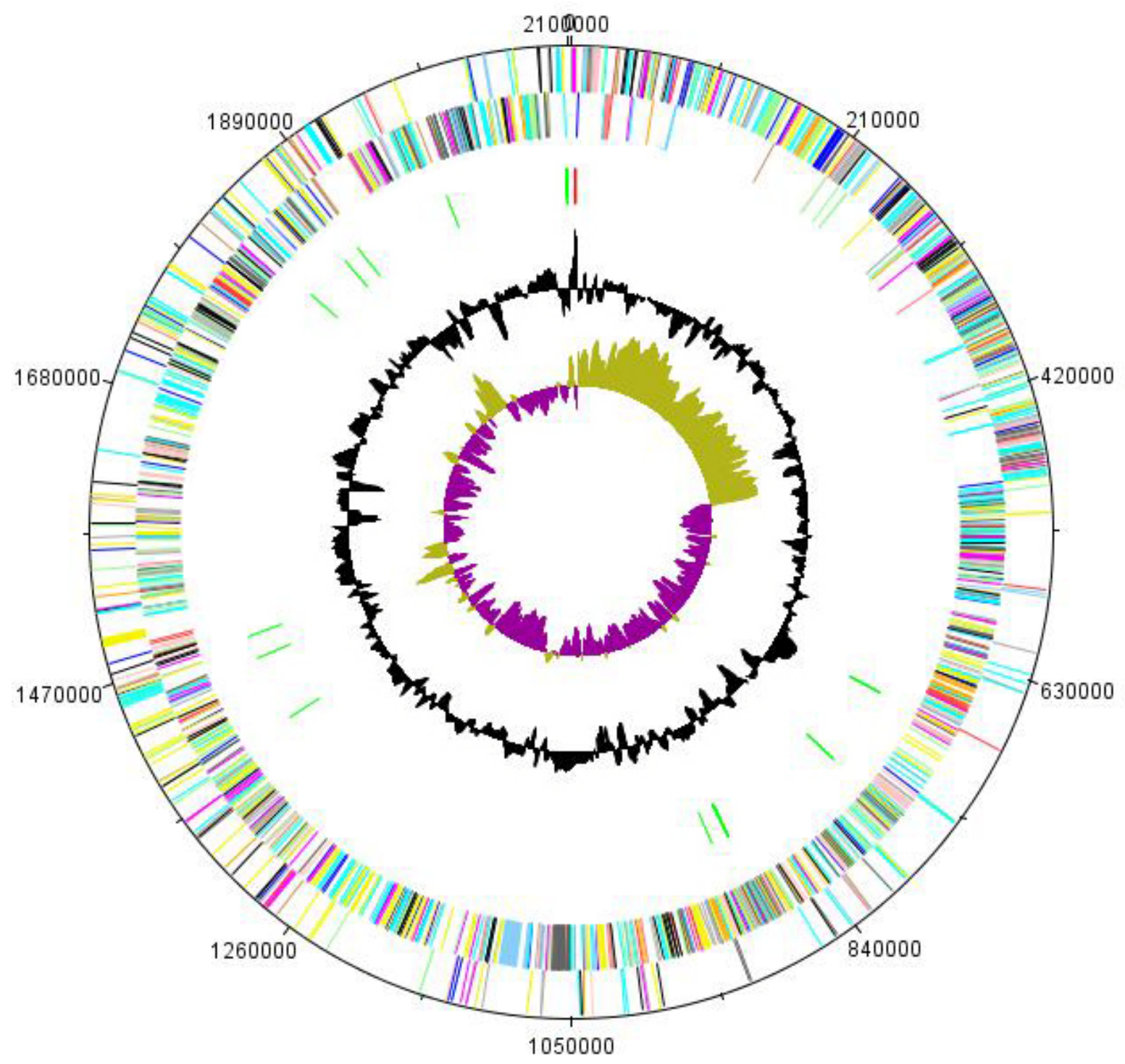

Figure 5. Graphical circular map of the chromosome. From the outside to the center: genes on the forward strand (colored by COG categories), genes on the reverse, RNA genes (rRNAs red, tRNAs green), G+C content, GC skew (purple negative values, olive positive values). 
Table 3. Nucleotide content and gene count levels of the genome

\begin{tabular}{lrr}
\hline Attribute & Value & \% of total \\
\hline Genome size (bp) & $2,101,866$ & \\
DNA coding region (bp) & $1,919,775$ & 91.34 \\
DNA G+C content (bp) & 712,533 & 33.9 \\
Number of replicons & 1 & \\
Extrachromosomal elements & 0 & \\
Total genes & 2,070 & 100 \\
RNA genes & 29 & 1.42 \\
rRNA operons & 1 & \\
Protein-coding genes & 2,041 & 98.59 \\
Genes with function prediction & 1,418 & 68.50 \\
Genes assigned to COGs & 1,439 & 69.52 \\
Genes with peptide signals & 128 & 6.18 \\
Genes with transmembrane helices & 542 & 26.18 \\
\hline
\end{tabular}

a The total is based on either the size of the genome in base pairs or the total number of protein coding genes in the annotated genome

Table 4. Number of genes associated with the 25 general COG functional categories

\begin{tabular}{crrl}
\hline Code & Value & \%age $^{\text {a }}$ & Description \\
\hline J & 137 & 6.71 & Translation \\
A & 0 & 0 & RNA processing and modification \\
K & 117 & 5.73 & Transcription \\
L & 130 & 6.36 & Replication, recombination and repair \\
B & 1 & 0.05 & Chromatin structure and dynamics \\
D & 20 & 0.98 & Cell cycle control, mitosis and meiosis \\
Y & 0 & 0 & Nuclear structure \\
V & 68 & 3.33 & Defense mechanisms \\
T & 60 & 2.93 & Signal transduction mechanisms \\
M & 63 & 3.09 & Cell wall/membrane biogenesis \\
N & 6 & 0.29 & Cell motility \\
Z & 0 & 0 & Cytoskeleton \\
W & 0 & 0 & Extracellular structures \\
U & 25 & 1.22 & Intracellular trafficking and secretion \\
O & 63 & 3.08 & Posttranslational modification, protein turnover, chaperones \\
C & 98 & 4.80 & Energy production and conversion \\
G & 47 & 2.30 & Carbohydrate transport and metabolism \\
E & 127 & 6.22 & Amino acid transport and metabolism \\
F & 57 & 2.79 & Nucleotide transport and metabolism \\
H & 54 & 2.64 & Coenzyme transport and metabolism \\
I & 38 & 1.86 & Lipid transport and metabolism \\
P & 90 & 4.40 & Inorganic ion transport and metabolism \\
Q & 20 & 0.97 & Secondary metabolites biosynthesis, transport and catabolism \\
R & 197 & 9.65 & General function prediction only \\
S & 143 & 7.0 & Function unknown \\
- & 602 & 29.49 & Not in COGs \\
\hline
\end{tabular}

${ }^{a}$ The total is based on the total number of protein coding genes in the annotated genome. 


\section{Comparison with genomes from other}

\section{Peptoniphilus species}

The genomes from only three validated Peptoniphilus species are currently available. Here, we compared the genome sequence of $P$. grossensis strain ph5 $5^{\mathrm{T}}$ with those of $P$. harei strain ACS-146-V-Sch2b, P. duerdenii strain ATCC BAA$1640, P$. lacrimalis strain $315-\mathrm{B}$, as well as $P$. timonensis strain $\mathrm{JC} 401^{\mathrm{T}}$ that we recently studied.

The draft genome sequence of $P$. grossensis strain ph5 ${ }^{\mathrm{T}}$ has a similar size to that of $P$. duerdenii $(2.10$ vs $2.12 \mathrm{Mb}$, respectively), but a larger size than $P$. lacrimalis, $P$. harei and $P$. timonensis $(1.69,1.83$ and $1.75 \mathrm{Mb}$, respectively). The $\mathrm{G}+\mathrm{C}$ content of $P$. grossensis is larger than $P$. lacrimalis and $P$. timonensis (33.9, 29.91 and $30.7 \%$, respectively) and comparable to $P$. duerdenii and P. harei $(34.24$ and 34.44, respectively). The gene content of $P$. grossensis is larger than those of $P$. duerdenii, $P$. lacrimalis, $P$. harei and $P$. timonensis $(2,041,1,988$,
$1,636,1,765$ and 1,922, respectively). The ratio of genes per MB of $P$. grossensis is larger to those of $P$. lacrimalis and P. harei (986, 968 and 964, respectively) and smaller to those of $P$. duerdenii and $P$. timonensis $(1,009$ and 1,111, respectively). However, the distribution of genes into COG categories was highly similar in all four compared genomes (Figure 6). In addition, $P$. grossensis shares a mean $82.0 \%$ (range $70-99 \%$ ), 85.8\% (range 70.7-100\%), 86.03 (range 70-100\%) and 87.78\% (range 70.8-100\%) sequence similarity with $P$. duerdenii, $P$. timonensis, $P$. harei and P. lacrimalis, respectively, at the genome level. On the basis of phenotypic, phylogenetic and genomic analyses, we formally propose the creation of Peptoniphilus grossensis sp. nov. which includes strain ph5 ${ }^{\mathrm{T}}$. This bacterium has been found in Marseille, France.

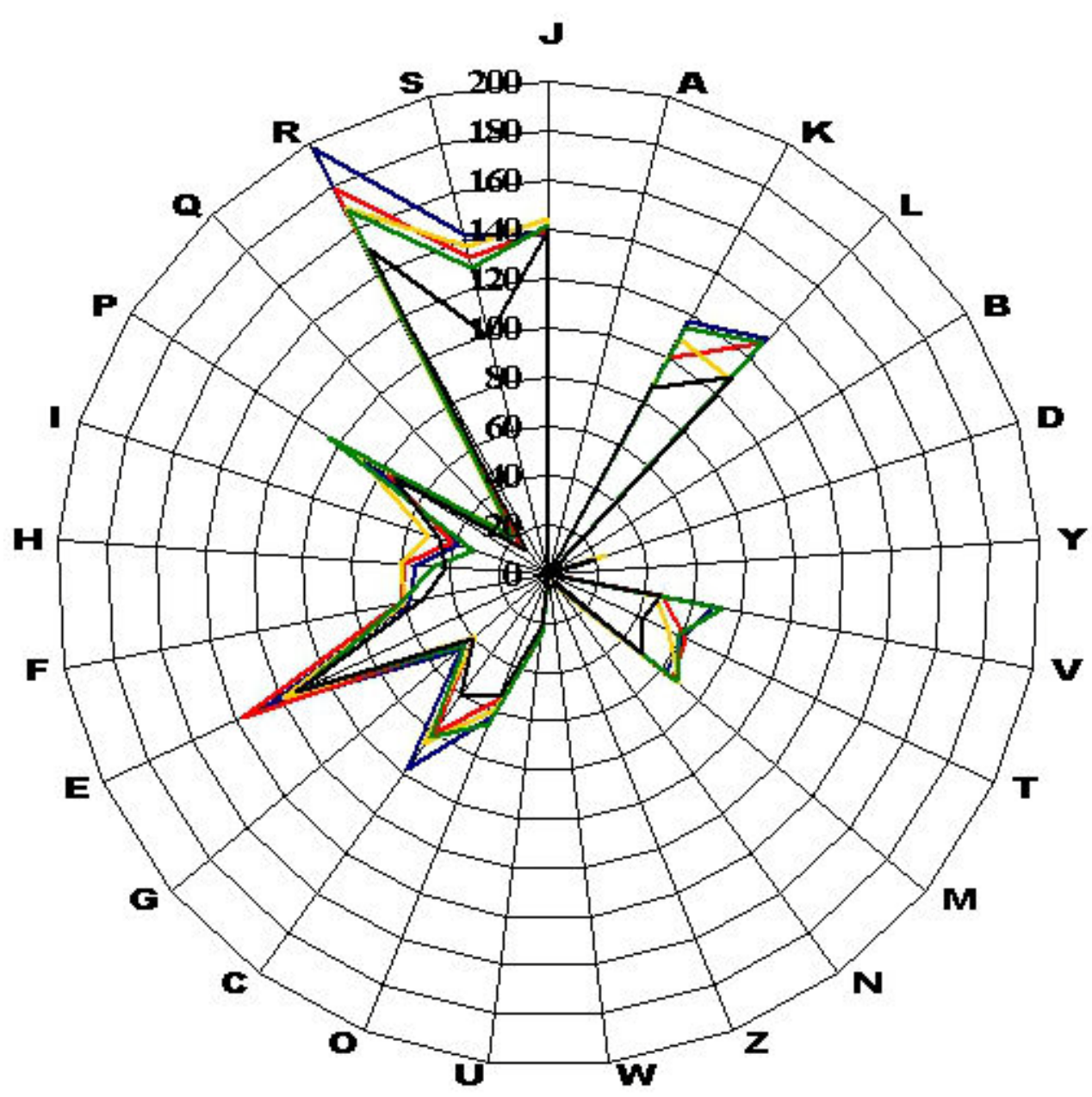

Figure 6. Compared distribution of predicted genes of $P$. grossensis (blue), $P$. timonensis (red), P. harei (yellow), P. duerdenii (green) and P. lacrimalis (black) into COG categories. 
Description of Peptoniphilus grossensis sp. nov. Peptoniphilus grossensis (gro.sen'sis. L. gen. masc. n. grossensis, of gros, the French adjective for fat, as the strain was isolated from an obese patient).

Colonies are $1 \mathrm{~mm}$ in diameter on blood-enriched Columbia agar and Brain Heart Infusion (BHI) agar. Cells are coccoid with a mean diameter of 1.2 $\mu \mathrm{m}$, occurring mostly in pairs, short chains or small clumps. Growth is only achieved anaerobically. The optimal growth temperature is $37^{\circ} \mathrm{C}$. Cells are Gram-positive, endospore-forming, and non-motile. Cells are negative for catalase and positive for indole production. Acid is produced from mannose. Positive reactions are observed for

\section{References}

1. Relman DA. Microbial Genomics and Infectious Diseases. N Engl / Med 2011; 365:347-357. PubMed http://dx.doi.org/10.1056/NEJMra1003071

2. Rossello-Mora R. DNA-DNA reassociation methods applied to microbial taxonomy and their critical evaluation. In: Stackebrandt E, ed. Molecular Identification, Systematics, and population Structure of Prokaryotes.Berlin: Springer, 2006:23-50.

3. Stackebrandt E, Ebers J. Taxonomic parameters revisited: tarnished gold standards. Microbiol Today 2006; 33:152-155.

4. Tindall BJ, Rosselló-Móra R, Busse HJ, Ludwig W, Kämpfer P. Notes on the characterization of prokaryote strains for taxonomic purposes. Int I Syst Evol Microbiol 2010; 60:249-266. PubMed http://dx.doi.org/10.1099/ijs.0.016949-0

5. Jousimies-Somer HP, Summanen DM, Citron EJ, Baron HM. Wexler, Finegold SM. WadsworthKTL anaerobic bacteriology manual, 6th ed Belmont: Star Publishing, 2002.

6. Murdoch DA, Mitchelmore IJ, Tabaqchali S. The clinical importance of gram-positive anaerobic cocci isolated at St Bartholomew's Hospital, London, in 1987. J Med Microbiol 1994; 41:36-44. PubMed http://dx.doi.org/10.1099/00222615-41$\underline{1-36}$

7. Ezaki T, Kawamura Y, Li N, Li ZY, Zhao L, Shu S. Proposal of the genera Anaerococcus gen. nov., Peptoniphilus gen. nov. and Gallicola gen. nov. for members of the genus Peptostreptococcus. Int J Syst Evol Microbiol 2001; 51:1521-1528. PubMed

8. Song Y, Liu C, Finegold SM. Peptoniphilus gorbachii sp. nov., Peptoniphilus olsenii sp. nov. arginine arylamidase, tyrosine arylamidase, histidine arylamidase and leucine arylamidase. Cells are susceptible to penicillin G, amoxicillin, ceftriaxone, cefalexin, imipenem, fosfomycin, erythromycin, doxycyclin, rifampicin, vancomycin, metronidazole, but resistant to ciprofloxacin and cotrimoxazole. The $\mathrm{G}+\mathrm{C}$ content of the genome is $33.9 \%$. The genome and 16 SrRNA sequences are deposited in GenBank under accession numbers CAGX00000000 and JN837491, respectively. The type strain ph5 ${ }^{\mathrm{T}}(=$ CSUR P184 = DSM 25475) was isolated from the fecal flora of an obese French patient.

and Anaerococcus murdochii sp. nov. isolated from clinical specimens of human origin. J Clin Microbiol 2007; 45:1746-1752. PubMed http://dx.doi.org/10.1128/JCM.00213-07

9. Rooney AP, Swezey JL, Pukall R, Schumann P, Spring S. Peptoniphilus methioninivorax sp. nov., a Gram-positive anaerobic coccus isolated from retail ground beef. Int I Syst Evol Microbiol 2011; 61:1962-1967. PubMed http://dx.doi.org/10.1099/ijs.0.024232-0

10. Field D, Garrity G, Gray T, Morrison N, Selengut J, Sterk P, Tatusova T, Thomson N, Allen MJ, Angiuoli SV, et al. The minimum information about a genome sequence (MIGS) specification. Nat Biotechnol 2008; 26:541-547. PubMed http://dx.doi.org/10.1038/nbt1360

11. Woese CR, Kandler O, Wheelis ML. Towards a natural system of organisms: proposal for the domains Archae, Bacteria, and Eukarya. Proc Natl Acad Sci USA 1990; 87:4576-4579. PubMed http://dx.doi.org/10.1073/pnas.87.12.4576

12. Gibbons NE, Murray RGE. Proposals Concerning the Higher Taxa of Bacteria. Int I Syst Bacteriol 1978; 28:1-6. http://dx.doi.org/10.1099/00207713-28-1-1

13. Garrity GM, Holt JG. The Road Map to the Manual. In: Garrity GM, Boone DR, Castenholz RW (eds), Bergey's Manual of Systematic Bacteriology, Second Edition, Volume 1, Springer, New York, 2001, p. 119-169.

14. Murray RGE. The Higher Taxa, or, a Place for Everything...? In: Holt JG (ed), Bergey's Manual of Systematic Bacteriology, First Edition, Volume 1, The Williams and Wilkins Co., Baltimore, 1984, p. 31-34. 
15. List Editor. List of new names and new combinations previously effectively, but not validly, published. List no. 132. Int J Syst Evol Microbiol 2010; 60:469-472.

http://dx.doi.org/10.1099/ijs.0.022855-0

16. Rainey FA. Class II. Clostridia class nov. In: De Vos P, Garrity G, Jones D, Krieg NR, Ludwig W, Rainey FA, Schleifer KH, Whitman WB (eds), Bergey's Manual of Systematic Bacteriology, Second Edition, Volume 3, Springer-Verlag, New York, 2009, p. 736.

17. Skerman VBD, Sneath PHA. Approved list of bacterial names. Int J Syst Bact 1980; 30:225-420. http://dx.doi.org/10.1099/00207713-30-1-225

18. Prévot AR. In: Hauderoy P, Ehringer G, Guillot G, Magrou. J., Prévot AR, Rosset D, Urbain A (eds), Dictionnaire des Bactéries Pathogènes, Second Edition, Masson et Cie, Paris, 1953, p. 1-692.

19. Ashburner M, Ball CA, Blake JA, Botstein D, Butler $\mathrm{H}$, Cherry JM, Davis AP, Dolinski K, Dwight SS, Eppig JT, et al. Gene ontology: tool for the unification of biology. The Gene Ontology Consortium. Nat Genet 2000; 25:25-29. PubMed http://dx.doi.org/10.1038/75556

20. Seng P, Drancourt M, Gouriet F, La Scola B, Fournier PE, Rolain JM, Raoult D. Ongoing revolution in bacteriology: routine identification of bacteria by matrix-assisted laser desorption ionization time-of-flight mass spectrometry. Clin Infect Dis 2009; 49:543-551. PubMed http://dx.doi.org/10.1086/600885

21. Prodigal. http://prodigal.ornl.gov

22. Benson DA, Karsch-Mizrachi I, Clark K, Lipman DJ, Ostell J, Sayers EW. GenBank. Nucleic Acids Res 2012; 40:D48-D53. PubMed http://dx.doi.org/10.1093/nar/gkr1202
23. Lowe TM, Eddy SR. t-RNAscan-SE: a program for imroved detection of transfer RNA gene in genomic sequence. Nucleic Acids Res 1997; 25:955-964. PubMed

24. Lagesen K, Hallin P, Rodland EA, Staerfeldt HH, Rognes T, Ussery DW. RNAmmer: consistent and rapid annotation of ribosomal RNA genes. Nucleic Acids Res 2007; 35:3100-3108. PubMed http://dx.doi.org/10.1093/nar/gkm160

25. Bendtsen JD, Nielsen H, von Heijne G, Brunak S. Improved prediction of signal peptides: SignalP 3.0. J Mol Biol 2004; 340:783-795. PubMed http://dx.doi.org/10.1016/j.jmb.2004.05.028

26. Krogh A, Larsson B, von Heijne G, Sonnhammer EL. Predicting transmembrane protein topology with a hidden Markov model: application to complete genomes. J Mol Biol 2001; 305:567580. PubMed http://dx.doi.org/10.1006/jmbi.2000.4315

27. Rutherford K, Parkhill J, Crook J, Horsnell T, Rice P, Rajandream MA, Barrell B. Artemis: sequence visualization and annotation. Bioinformatics 2000; 16:944-945. PubMed http://dx.doi.org/10.1093/bioinformatics/16.10.94 $\underline{4}$

28. Carver T, Thomson N, Bleasby A, Berriman M, Parkhill J. DNAPlotter: circular and linear interactive genome visualization. Bioinformatics 2009; 25:119-120. PubMed http://dx.doi.org/10.1093/bioinformatics/btn578

29. Darling AC, Mau B, Blattner FR, Perna NT. Mauve: multiple alignment of conserved genomic sequence with rearrangements. Genome Res 2004; 14:1394-1403. PubMed http://dx.doi.org/10.1101/gr.2289704 